\title{
SIGNAL ANALYSIS ON SOIL STRESS FROM VIBRATING COMPACTION BASED ON WAVELET TRANSFORM
}

\author{
ZHANG QING-ZHE ${ }^{1}$, YAN BING $^{2}$, DAI JING-LIANG ${ }^{3}$, YANG BAO-GUI ${ }^{4}$
}

\begin{abstract}
The paper presented the wavelet transform method for de-noising and singularity detection to soil compressive stress signal. The study results show that the reconstruction signals by the wavelet de-noising keeps the low frequency component at $[0,31.25 \mathrm{~Hz}]$ of the original signal and improves the high frequency property at other frequency bands. The impaction time from the start time to resonance time of the stress signals is varies with the depth of the soil. With the increase of times of compaction, the impaction time of the stress is decreasing in every layer. But the speed of reaching compacted status in each layer is different.
\end{abstract}

Keywords: Soil, Compressive Stress Signal, Wavelet Transform, De-Noising, Singularity Detection

\section{INTRODUCTION}

The compressive stress signal of soil during vibrating compaction is an unstable and transient saltation accompanied by strong noise. The common method of analyzing soil stress is analyzing its spectral characteristic through Fourier transform on the signal [1]. While, Fourier transform can not segregate signal from noise effectively. Otherwise, Fourier transform is a Global transformation without temporal resolution [2]. In order to analyze the short-time signal, the short-time Fourier transform could be a proper method. But the Time-Frequency Window of the short-time Fourier transform is unalterable, the temporal frequency resolution ratio would not change during analyzation according to the self-adaption of signal characteristic [3].

Wavelet Transform is a novel signal analysis method in recent years [3], [4]. Wavelet Transform has the alterable part Time-Frequency Window, so it can make use of Time-Frequency Window of different resolution ratio to "observe" images, which is honored as mathematic microscope of auto zoom and is suitable for analyzing the transient saltation vibration signal with strong noise, and the signal catastrophe point can

1 (Corresponding Author) Key Laboratory for Highway Construction Technique and Equipment of Ministry of Education of China,Chang'anUniversity,Xi'an710064,e-mail:zqzh@chd.edu.cn

2 Chang'an University, Xi'an 710064,e-mail:108714055@qq.com

3 Key Laboratory for Special Area Highway Engineering of Ministry of Education of China, Chang'an University, Xi'an 710064

${ }^{4}$ Chang'an University, Xi'an 710064 
be detected [3].With the increasingly improvement of wavelet theory and its deepening study, Wavelet Transform is widely used in signal analysis in every field [5], [6], [7]. In the field of civil engineering, Many scholars research on methods of de-noising and singularity detection based on wavelet transform to transient vibration test signals [8], [9], [10], [11]. The results show that wavelet transform has a lot of advantages in the aspect of signal detection and maintains both the smoothness and similarity of the vibration signal after de-noising. Meanwhile, it plays a key role in singularity detection of signals and its results far exceed the traditional Fourier transform. The above documents analyze vibration acceleration signal or the transient signal of soil compactness (Acceleration, Force) in the process of vibrating compaction by using the Wavelet transform, but there is no literature report on analysis of soil compressive stress signal during vibrating compaction.

To study the joint time-frequency property of soil compressive stress signal and the distribution and transformation law of the stress in the soil, so as to reveal the mechanism of the vibrating compaction, the paper presented the wavelet transform method for de-noising and singularity detection to soil compressive stress signal. Firstly, extract the stress signal of useful frequency band by wavelet transform to realize the effective segregation between signal and noise. Then, analyze the sequence signal of wavelet high frequency coefficients by wavelet singularity detection to detect the start time and resonance time of the compressive stress. The distribution and transformation law of the stress in the soil can be obtained by analyzing the impaction time from start time to resonance time. This paper has important theoretical significance to supplement and perfect the vibrating compaction theory and guide the engineering practice.

\section{SOIL VIBRATING COMPACTION}

The vibration compaction test of soil was implemented in the large soil tank of Key Laboratory for highway construction technique and equipment of ministry of education, Chang'an University. The test equipment uses a self-designed vibratory roller model [12]. The compaction test section length is $8 \mathrm{~m}$, width is $1 \mathrm{~m}$. In view of the vibratory roller that used in compacting and test condition, with nominal amplitude, vibration frequency and running speed of vibratory roller as the main factors, and use 3 factors and 3 levels of orthogonal working conditions, finally, these nine tests fully reflect the various factors on the test results. In each working condition, first static pressure 2 times, then turn the vibration compaction 12 times [13].

Before Test, turned loose soil to $35 \mathrm{~cm}$ in soil tank by hand, and watering in order to achieve the appropriate moisture content. Second, three dynamic strain gauge pressure cell were placed on 3 layers under the loess, and the depth from the soil surface were $5 \mathrm{~cm}, 15 \mathrm{~cm}$ and $25 \mathrm{~cm}$. When tested, with the DEWE-2010 data acquisition device records the soil compressive stress signals of the pressure cell during the vibration compaction process, it can capture the stress signal to the oscilloscope in real time, data 
storage and data processing. The block diagram acquisition system as shown in figure1. The sample frequency of soil compressive stress signal in the test is $2000 \mathrm{~Hz}$.

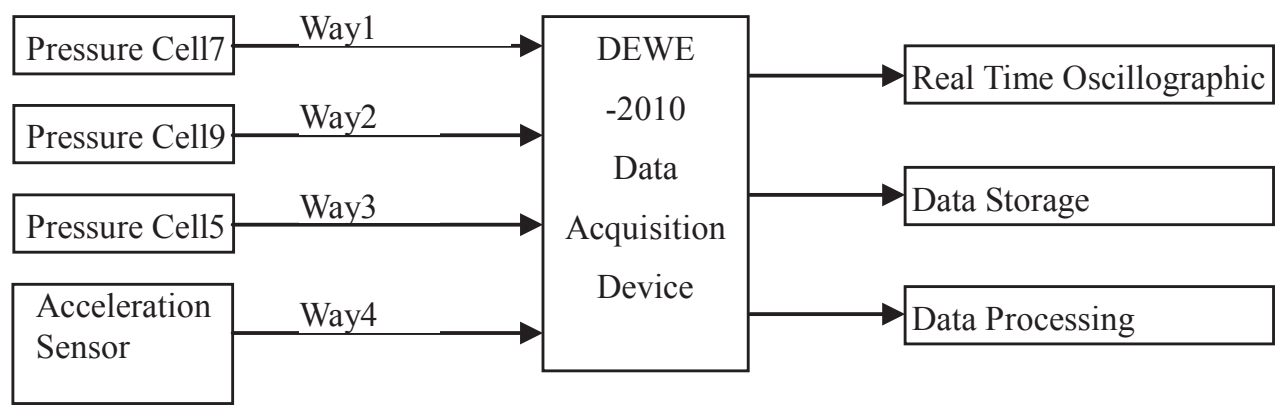

Fig. 1. Data Acquisition System

3. Signal analysis PRINCIPLe on WaVelet transform [3]

Let $\psi(t) \in L^{2}(R), \hat{\Psi}(\omega)$ is the result of the Fourier transform of $\psi(t)$. If $\hat{\Psi}(\omega)$ meets the admissible condition: $\left.C_{\Psi}=\int_{-\infty}^{\infty}|\Psi \hat{(\omega)}|^{2} /|\omega|\right) d \omega<\infty$, and call $\psi(t)$ basic wavelet function. Scale transformation and translation for the basic wavelet function, then a group of wavelet functions can be obtained.

$$
\psi_{a, b}(t)=\frac{1}{\sqrt{|a|}} \psi((t-b) / a) ; a, b \in R, a \neq 0
$$

In the formula (3.1), ' $\mathrm{a}$ ' is the scale factor which controls time window width of wavelet function, and ' $b$ ' is the displacement factor which controls translation of wavelet function on the time axis. The bigger the $|a|$ is, the wider the time window is and the narrower the frequency window is. It is can be proved that the product of time window width and frequency window width of wavelet function is constant.

Dyadic wavelet transform is used widely in practice. Let $a=2^{j}, b=2^{j} \cdot k$, a group of dyadic wavelet functions can be obtained.

$$
\psi_{j, k}(t)=2^{-\frac{j}{2}} \psi\left(2^{-j} t-k\right) ; j, k \in Z
$$

The wavelet transform of signal $f(t)$ is defined as: 


$$
W_{f}(j, k)=<f(t), \psi_{j, k}(t)>=\frac{1}{2^{j}} \int_{-\infty}^{\infty} f(t) \cdot \psi\left(2^{-j} t-k\right) d t
$$

From the perspective of signal processing, the changes of ' $a$ ' are equivalent to a continuous change of transmission bands of band-pass filter. And changes of ' $b$ ' are equivalent to the band-pass filtering to signal at different time. By changing the ' $a$ ', signal can be observed through wavelet transform on wide time window (that is narrow frequency window) at low frequency, but on narrow time window (that is wide frequency window) at high frequency. The local time-frequency characteristics of wavelet transform is very suitable for analysis on signal that with slow change at low-frequency but with quick change at high-frequency.

\subsection{Principle on WAVelet transforming MEthod FOR DE-NOISING}

the soil compressive stress signal during vibrating compaction is unstable and transient saltation signals accompanied by strong noise. The noise can be eliminated effectively by taking advantage of local time-frequency characteristics of wavelet transform, so as to extract ideal stress signals. The fundamental principle of wavelet transform for de-noising is letting some high-frequency components be 0 selectively, and retaining some useful frequency band, then reconstruct the signal by wavelet reconstruction algorithm.

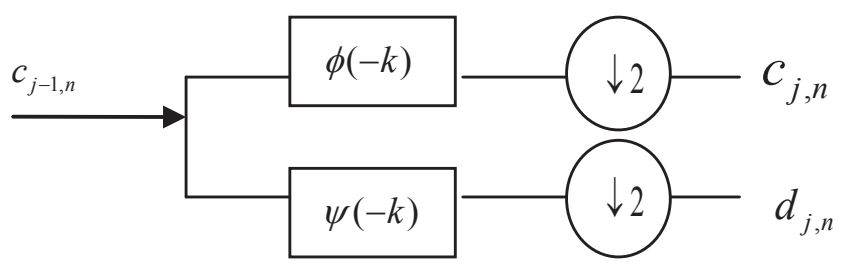

Fig. 2. Multi-resolution wavelet decompositions

Mallat [4] proposed multi-resolution wavelet analysis when he was constructing orthogonal wavelet basis. Let $\psi(n)$ is a wavelet function, corresponds to band-pass filter, and $\phi(n)$ is the scaling function that corresponds to $\psi(n)$, that is a low-pass filter. From the perspective of multi-resolution analysis, wavelet decomposition is equivalent to make the low-frequency signal $C_{j-1, n}$ with scale $=j-1$, through the band-pass filter $\psi(-k)$ and a low-pass filter $\phi(-k)$ respectively. Then after downsampling, we can get a low-frequency signal $C_{j, n}$ and a high-frequency signal $d_{j, n}$ with the same scale $=j$. The principle of multi-resolution wavelet decompositions is shown as Figure 2. 
The low-frequency signal can be decomposed step by step by multi-resolution wavelet analysis. In every decomposition, signal can be divided into low-frequency band and high-frequency band. Let the occupied frequency band of original signal be $\left[0, f_{\max }\right]$, then the signal can be divide into low-frequency band and high-frequency band which frequency range is $\left[0, f_{\max } / 2^{k}\right]$ and $\left[f_{\max } / 2^{k}, f_{\max } / 2^{k-1}\right]$ respectively in the $k$-th wavelet decomposition.

Signal can be decomposed into different frequency bands through many multi-resolution wavelet decompositions. By extracting the useful frequency band but suppressing the high frequency band of signal, and making use of wavelet reconstruction algorithm [3], we can obtain the de-noising compressive stress signal.

\subsection{THE PRINCIPLE ON WAVELET TRANSFORMING SINGULARITY DETECTION}

The singular point often corresponds to catastrophe point of amplitude or frequency of signal, which is one of signal's characteristics. The change of local modulus maximum of wavelet transform coefficient at multi-scale represents singular point of signal [2], so making use of the wavelet transform can detect the singular value of signal [3], [14], [15]. The principle of singularity detection is decompose signal at multi-resolution spatial by wavelet transform. In singular point of signal, high frequency coefficient of wavelet transform at different levels has local modulus maximum, the time that singular point appears can be determined by detecting local modulus maximum.

To detect the singular value of signal effectively, the chosen wavelet function should has good regularity. So wavelet function must be the first or second order derivative of a certain low-pass function. Because wavelet transform under different scales is equivalent to observing signal by filters with different frequency band. Only under the suitable scale, can wavelet transform caused by every catastrophe point avoid interference. Therefore, we need to synthesize results of multi-scale detection to detect singular value of signal in practice.

\section{WAVELET ANALYSIS ON STRESS SIGNAL OF SOIL}

The soil compressive stress signal recorded from vibrating compaction inevitably contains all kinds of noises. Noise is a kind of high-frequency saltation signal. In order to eliminate noise interference in singularity detection, the original compressive stress with noisy signals should be de-noising by wavelet transform method firstly. Then detect the singular value of the de-noising signals to work out the impaction time from start to resonance of the stress signals, so as to analyze the distribution and transformation law of the stress in the soil under different test conditions.

In this section, the soil compressive stress signal under working condition 2 is used to analyze by wavelet transform method. The vibrating compaction operating param- 
eters are that vibration frequency is $30 \mathrm{~Hz}$, nominal amplitude is $1.2 \mathrm{~mm}$, and running speed is $0.8 \mathrm{~km} / \mathrm{h}$ in this working condition.

\subsection{DenOISING OF WAVELET TRANSFORMING ON ORIGINAL STRESS SIGNAL OF SOIL}

The soil compressive stress signal during vibrating compaction is an unstable and transient saltation signal. Sym8 orthogonal symlet wavelet function is used to analyze the stress signal in this paper [14]. Because the vibration frequency of vibratory roller is $30 \mathrm{~Hz}$ under this working condition, theoretically, the soil compressive stress appears resonance peak at about $30 \mathrm{~Hz}$. So the signal within this frequency range is useful, which should be retained.

According to sampling theorem, the analysis frequency of wavelet transform should be a half of sample frequency, that is $[0,1000 \mathrm{~Hz}]$. Decompose the original compressive stress signal $\mathrm{s}(\mathrm{t})$ by sym 8 orthogonal wavelet function to five level. Based on the frequency band segregation theorem of wavelet decomposition, the occupied frequency of low-frequency signal at the fifth level is $[0,31.25 \mathrm{~Hz}]$, whose signal should be retained totally. The occupied frequency bands of high-frequency signals at other different levels are respectively $\left[1000 / 2^{k}, 1000 / 2^{k-1}\right] \mathrm{Hz}$, and here, $\mathrm{k}=1, \ldots 5$, which means the level of wavelet decomposition. Dispose high frequency coefficient at different levels by method that choosing soft threshold according to heuristic threshold and making use of the estimated noise at every level adjust threshold. Then making use of the disposed high frequency coefficient and low-frequency signal at the fifth level reconstruct signal by wavelet reconstruction algorithm.

Figure 3(a) shows the original stress signals of soil. Figure 3(b) shows the de-noising signals by low-pass filtering in the frequency domain, and the cut-off frequency of filter is $32 \mathrm{~Hz}$. Figure 3(c) shows the de-noising signals by wavelet transform. According to the comparison result, the traditional low-pass filtering cause the reconstruction signal amplitude distortion and eliminate all frequency components that are above 32 Hz. While wavelet transform not only eliminate the noise but also retain the signal characteristics very well in the meantime.

Figure 4(a) and 4(b) respectively shows the five level decomposed signals in every size by $\mathrm{db} 5$ wavelet function to the signals of the original compressive stress $\mathrm{s}(\mathrm{t})$ and de-noising signal sd(t). Comparing high-frequency band of the two decomposed signals at every level, it can be seen clearly that de-noising signal by wavelet decomposition keeps the low frequency (the frequency is $[0,31.25 \mathrm{~Hz}]$ ) characteristic of the original soil compressive signal. And at the same time, it eliminates the noise from other high frequency band and improves the characteristic of high frequency signal. 


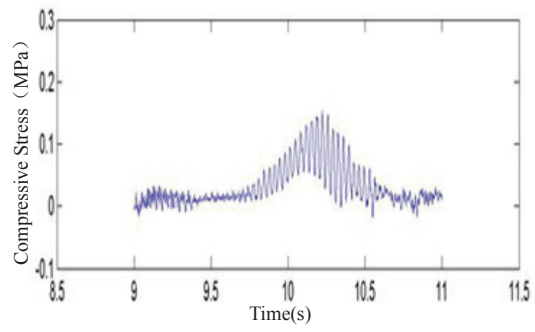

(a) Original stress signal of soil

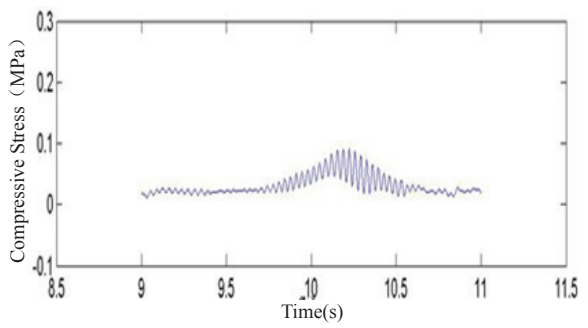

(b) De-noising signals after low-pass filtering in the frequency domain

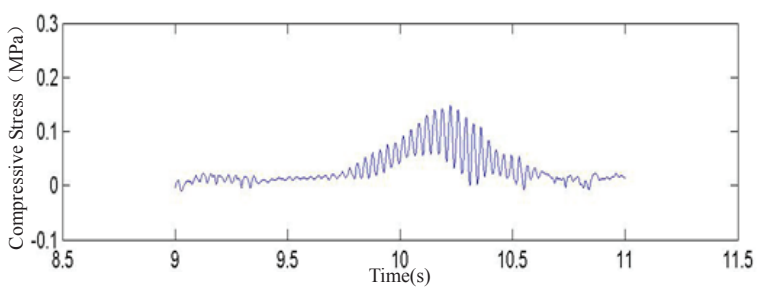

(c)De-noising signals after wavelet transform

Fig. 3. Original compressive stress signals and de-noising signals

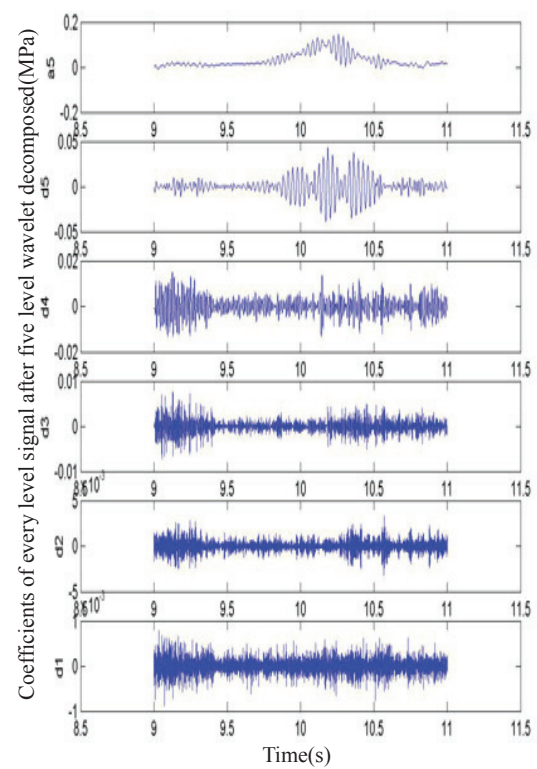

(a)Wavelet decomposition of original stress signal s (t)

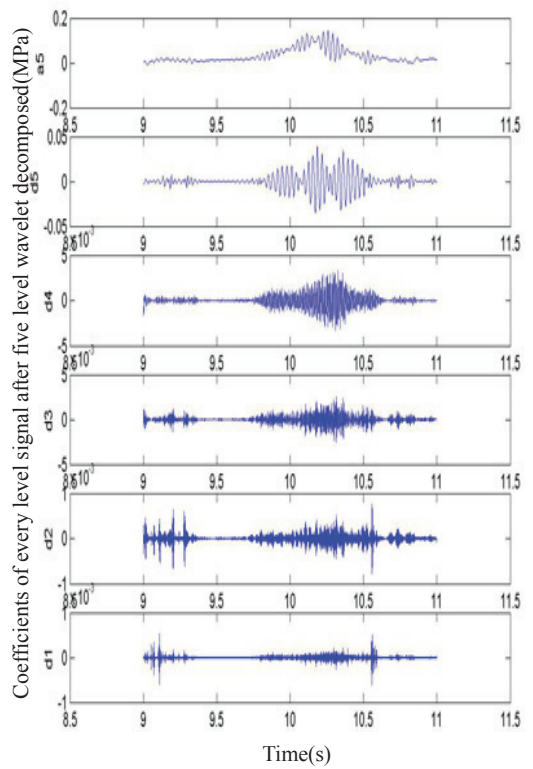

(b) Wavelet decomposition of de-noising signal $s d(t)$

Fig. 4. Comparison of coefficients of every level signal after five level wavelet decomposed 


\subsection{Singularity DeteCtion OF WAVELET tRANSFORM ON SOIL COMPRESSIVE STRESS SIGNAL}

Combining characteristics of compressive stress signal and vibrating compaction test, it can be known that there are two singular values in the sequence diagram of soil compressive stress signal. The first singular point is the start point of the stress, corresponds to the first local modulus maximum of wavelet high frequency coefficient in the timing sequence. The second singular point is peak point of the stress where signal amplitude enlarges which is caused by resonance suddenly generates, corresponds to modulus maximum of wavelet high frequency coefficient in the timing sequence. Detecting these two feature points, the impaction time from start to resonance of the stress signals can be worked out. This is significant to analyze the distribution and transformation law of the stress in the soil and reveal the mechanism of the vibrating compaction.

Here, we choose db5 wavelet function (which has better regularity) analyze compressive stress signal by Multi-resolution wavelet method [15]. According to the former analysis, decompose the de-noising signal $\mathrm{sd}(\mathrm{t})$ to five level. The wavelet coefficients at every level after decomposition is shown in Figure 4(b).

To extract local modulus maximum of wavelet coefficient more precise, Hilbert transform can be used to extract the envelope in every level wavelet coefficients [16]. The envelope figure of wavelet coefficient is shown in Figure 5. Considering the positions of three pressure sensors buried in every layer are not absolutely located in a perpendicular line, which may cause the inconsistency of start and resonance time in different soil layers. To eliminate the influence, the impaction time from start to resonance time of the stress signals should be analyzed.

Using the above method, analyze the soil compressive stress signal in top, middle, and bottom layer during second compaction under working condition 2. Combining the local modulus maximum of high-frequency signal at every level, the start time, resonance time and the impaction time of the soil compressive stress signal in every layer can be worked out and which are shown in Table 1 .

Table 1.

The impaction time of the soil compressive stress signals based on singularity detection of wavelet transform

\begin{tabular}{|c|c|c|c|}
\hline Sensor Location & The start time of signal & The resonance time of signal & The impaction time \\
\hline The top soil & $9.9135 \mathrm{~s}$ & $10.2871 \mathrm{~s}$ & $0.3736 \mathrm{~s}$ \\
\hline The middle layer & $9.9024 \mathrm{~s}$ & $10.3119 \mathrm{~s}$ & $0.4095 \mathrm{~s}$ \\
\hline the bottom soil & $9.9173 \mathrm{~s}$ & $10.3671 \mathrm{~s}$ & $0.4498 \mathrm{~s}$ \\
\hline
\end{tabular}




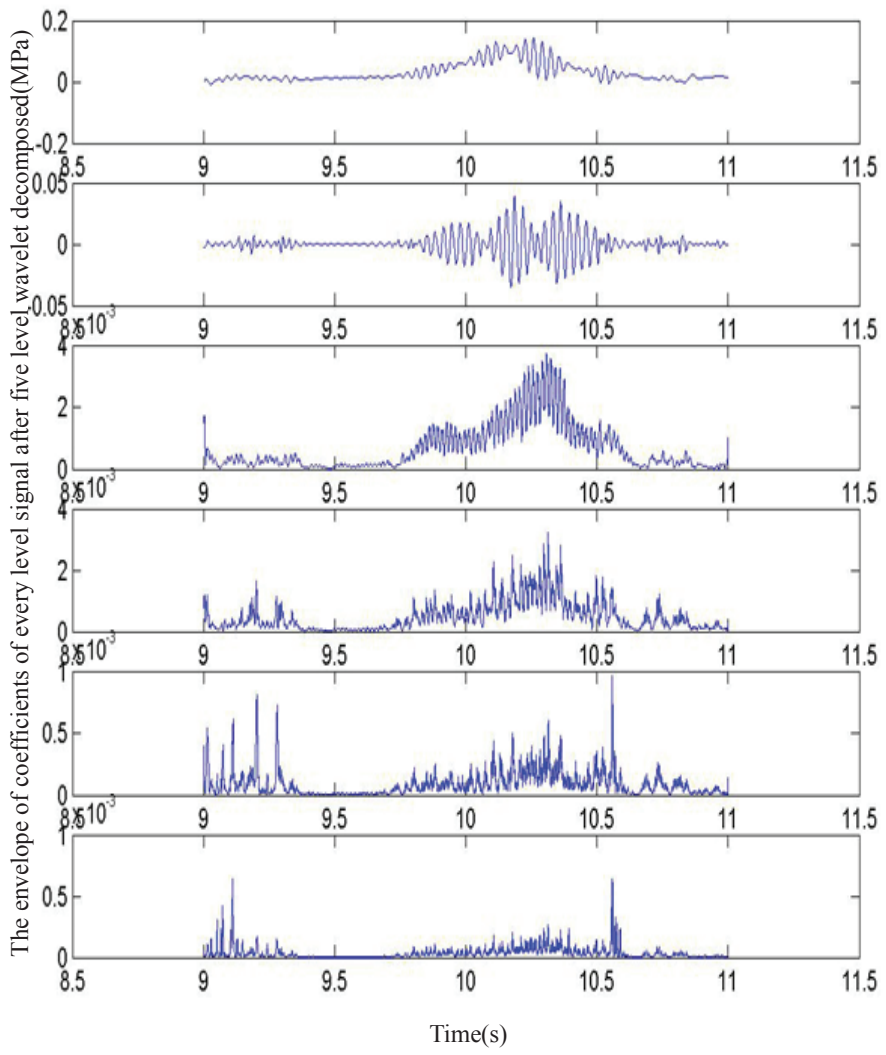

Fig. 5. The envelope figure of wavelet coefficient extracted by Hilbert transform

The analysis result shows that the impaction time of soil compressive stress is various at different depths of soil. The impaction time of the stress on the top soil is shortest, and vibrating energy absorbed by top soil is fastest. Middle layer takes the second place, and bottom soil takes the third place.

\subsection{ANALYSIS ON SOIL STRESS SIGNAL UNDER WORKING CONDITION 2}

According to the above theorems, the section analyze soil compressive stress signals under working condition 2 during all the times of compaction by wavelet de-noising and singularity detection, and detect the start time and resonance time of soil stress in every layer so as to work out the impaction time from start to resonance.

Figure 6 is the relation curve between the impaction time of the stress signals in every layer and the times of compaction. Figure 6(a) shows the impaction time curve of forward rolling process (in first, third, fifth, seventh, ninth, eleventh times). Figure 
6(b) shows the impaction time curve of reserve rolling process (in second, fourth, sixth, eighth, tenth, twelfth times). Analyzing Figure 6, it can be seen that: (1) During every compaction, the impaction time of the stress on the top soil is shortest, the middle layer takes the second place and the bottom soil takes the third place. (2) With the increase of times of compaction, the impaction time of the stress decreases in every layer. The impaction time of the stress on the top soil is shortest and it shows that the top soil arrives to its compacted status at the fastest speed. The middle layer takes the second place, the bottom soil takes the third place which shows that it arrives to its compacted status at a slower speed.

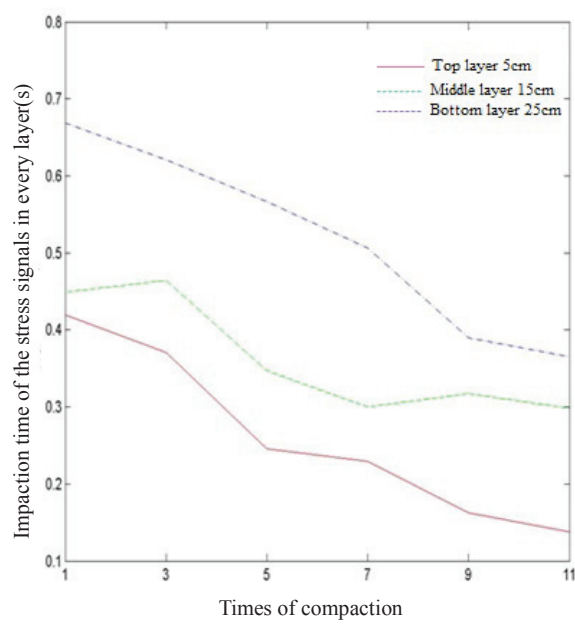

(a) Forward rolling

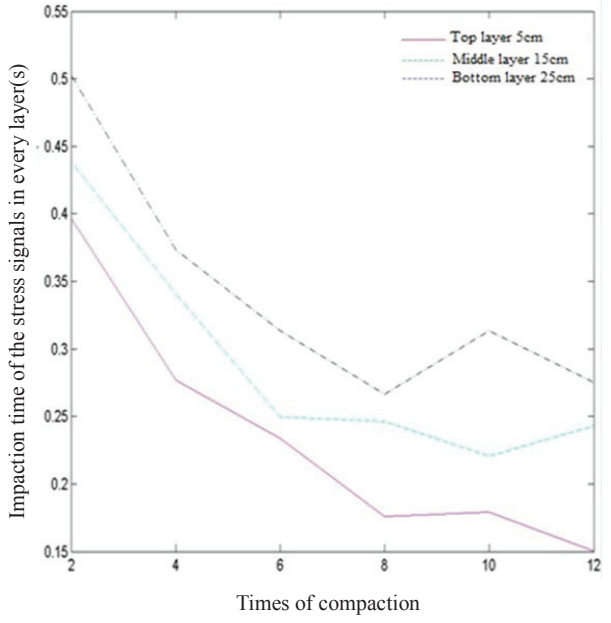

(b) Reverse rolling

Fig. 6. The Relation curve between the impaction time of the stress signals in every layer and the times of compaction

\section{ConClusion}

According to the above analyses, it can be concluded as the following:

(1) The paper reconstruct ideal stress signal of soil by wavelet transforming de-noising. The de-noising stress of soil retains the low frequency characteristic of original signal at $[0,31.25 \mathrm{~Hz}]$ frequency band, and in the meantime it improves the characteristic of other high-frequency signal.

(2) The paper detect the start time and resonance time of soil compressive stress by singularity detection based on wavelet transform, and work out the impaction time from start to resonance. The results show that the impaction time from the start to resonance time of the stress signals is different with the depth of the soil. The 
impaction time on the top soil is shortest, and the vibrating energy absorbed by the top soil is fastest. The middle layer takes the second place and the bottom soil takes the third place.

(3) With the increase of times of compaction, the impaction time of the stress is decreasing in every layer, this indicates that the soil compactness increases, but the speed of reaching compacted status in each layer is different. The impaction time of the stress on the top soil is shortest and it shows that the top soil arrives to its compacted status at the fastest speed. The middle layer takes the second place and the bottom soil takes the third place. It shows that the bottom soil arrives to its compacted status at a slower speed.

(4) Increase the times of compaction, the compactness of every layer in soil will increase. When it comes to a certain times of compaction, the compactness of top soil will be unchangeable and the energy is almost absorbed by deeper soil layer. The energy passes down from the top layer at this moment, and compactness of every layer will increase in turn then get their compacted status.

\section{Acknowledgments}

The Project was Supported by the Special Fund for Basic Scientific Research of Central Universities, Chang'an University(CHD2011JC073)and National Natural Science Foundation of China (11202036).

\section{REFERENCES}

1. Alan V. Oppenheim, Alan S. Willsky, With s. Hamid Nawab, Signals and Systems (The Second Edition).

2. Lu Wen-Xiang, Du Run-sheng, Mechanical Engineering testing, Information, Signal Analysis (The Second Edition), Huazhong University of Science Press, 1999.

3. Cheng Zheng-XIng, The Wavelet Analysis Algorithm and Application, Xi 'an jiaotong University Press, 2000.

4. S. G. Mallat, Multi-frequency Channel Decompositions Of Images And Wavelet Models, IEEE Transactions on Acoustics, Speech and Signal Processing, 37(12): 2091- 2110, 1989

5. Chang Yun-hua, Li Zong-wei, Yin Da-Juan, Non-stationary Vibration Signals Of The Wavelet Analysis Method, Noise And Vibration Control,5, 98-101, 2008.

6. Li Jian-feng, Shi Guang-Fu, Zhang Ping, Noise-reducing of vibration signal in SRM ground test by using wavelet analysis, Journal of Solid Rocket Technology, 25(4), 63-66, 2002

7. Wang Nan, Du Jin-song, Wavelet De-noising In The Application Of Vibration Signal Processing, Chinese Journal of Scientific Instrument, 22(4), 225-226, 2001.

8. YI FEI, Vibratory Roller Vibration Aceeleration Synchronous Detection and Data Processing, Journal of Chongqing Jiaotong University, 30(1), 162-165, 2011.

9. Li ZHe, The Mechanism of B Group Filling Subgrade Compaction and Wavelet Analysis of Dynamic Monitoring,Beijing: Beijing Jiaotong University, 2010.

10. Jin Jian-ming, Wang Kui-hua, Bu Fa-dong, Xie Kang-He, Multi-resolution Wavelet analysis on compactness measurement of soft soil by instant hammering method, Chinese Journal of Rock Mechanics and Engineering, 23(13), 2271-2275, 2004. 
11. Ju CAI-Mei, In-Vehicle Compaction Meter, Xi'an, Chang'an University, 2001.

12. Yao Yun-shi, Xiao Gang, Dong Xiu-hui, Feng Zhong-Xu, Experimental Study of Doubl-e Frequency Composed Vibrating Compaction, China Journal of Highway and Transport, 19(1), 122-126, 2006.

13. PRC National Standard,Vibratory Roller Performance Test Methods (GB4478-1995, China Standards Press, 1995.

14. Yu fu, Wen Sheng-ti, Guo Hua-xu, Wavelets and Singularities In Bearing Vibration Signals, Proceedings of the Second International Conference on Machine Learning and Cybernetics, Xi'an, 2433-2436, 2003.

15. W. L. Hwang, S. G. Mallat, Singularities And Noise Discrimination With Wavelets, 1992 IEEE International Conference on Acoustics, Speech, and Signal Processing, 4, 377-380, 1992.

16. Lei Ji-Rao, He Shi-DE, Engineering Processing Technology, Chongqing University Press, 96-97, 1990.

Received: 02.12.2013

Revised: 10.06 .2014 